\title{
LA RELACIÓN ENTRE LUDIFICACIÓN Y PRIMERA INFANCIA DESDE LA PERSPECTIVA DEL APRENDIZAJE. UN ESTADO DEL ARTE
}

The relationship between early childhood from the perspective of learning and gamification. A state of the art

A relação entre a primeira infância na perspectiva da aprendizagem e gamificação. Um estado da arte

\section{Edgar Oswaldo Pineda Martínez}

Universidad Santo Tomas, Sede Villavicencio. Grupo de Investigación A.B.A. Telf.: 3213616009. Correo electrónico: edgarpin535@gmail.com

\section{Paula Andrea Orozco Pineda}

Corporación Universitaria Minuto de Dios UNIMINUTO, Vicerrectoría Regional Llanos. Grupo de Investigación Trabajo de Llano. Telf.: 3134307788 Correo electrónico: paulaorozpi@gmail.com

\section{Resumen}

Este artículo pretende ofrecer un estado del arte actualizado sobre el abordaje del concepto de ludificación en literatura especializada sobre el aprendizaje en la primera infancia. Para tal efecto, expone la revisión de documentos investigativos sobre los conceptos de ludificación y gamificación que hayan sido publicados en revistas especializadas y que previamente hayan tenido revisión por pares académicos o comités científicos. Para este fin, se establece que el parámetro de revisión será el carácter motivacional para el uso o aplicación de estrategias de ludificación. Asimismo, el articulo expone resultados de la revisión de la literatura desde cuatro variables dependientes (aprendizaje, infancia, motivación, conducta). La revisión permitió identificar como las estrategias basadas en ludificación proporcionan efectos positivos y cambios conductuales y motivacionales de los sujetos que son dependientes del contexto donde es aplicado.

Palabras clave: gamificación, ludificación, revisión documental, análisis bibliográfico. 


\begin{abstract}
This article aims to give a state of the art update on the approach to the concept of gamification in literature specializing on learning in early childhood. To do so, it exposes review research documents about the concept of gamification which have been published in specialized magazines and who have previously had a review by academic peers or scientific committees. For this purpose, sets that the revision parameter will be the motivational character for the use or application of strategies of gamification. Also, the article presents results of the review of the literature from four dependent variables (learning, childhood, motivation, behavior). The review identified as gamification based strategies provide positive effects and behavioral changes and motivational subjects who are dependent upon the context where is applied.
\end{abstract}

Key Words: gamification, documents revision, bibliographic analysis.

\title{
Resumo
}

Este artigo pretende dar uma actualização do estado da arte sobre a abordagem ao conceito de Gamificação na literatura especializada sobre o aprendizado na primeira infância. Para fazer isso, ele expõe documentos de pesquisa de revisão sobre os conceitos de ludificacion e gamificação que foram publicados em revistas especializadas e que anteriormente tiveram uma revisão por pares acadêmicos ou comités científicos. Para esta finalidade, define que o parâmetro de revisão será o caráter motivacional para a utilização ou aplicação de estratégias de Gamificação. Além disso, o artigo apresenta os resultados da revisão da literatura de quatro variáveis dependentes (aprendizagem, infância, motivação, comportamento). A revisão identificada como estratégias baseadas em ludificacion proporcionam efeitos positivos e alterações comportamentais e motivacionais indivíduos que são dependentes do contexto onde é aplicado.

Palavras-chave: Gamificação, revisão, análise bibliográfica

\section{Introducción}

Durante las últimas dos décadas, la primeria infancia y sus componentes y factores asociados se han posicionado de forma significativa en la agenda de las políticas públicas en Colombia. Los anterior, es consecuencia de la adopción y cumplimiento de los compromisos emanados de la Constitución Política de Colombia (1991), de la Convención de los Derechos del Niño (1989), y del Código de la Infancia y la 
Adolescencia colombiano (Ley 098 de 2006), documentos que velan por hacer efectivo el principio de la prevalencia y prioridad de los derechos de los niños y niñas en sus respectivos contextos y territorios.

Los diferentes avances en la definición de una Política Pública a partir de un marco de referencia común, han permitido aunar esfuerzos para lograr proyectos de carácter integral que propendan por la mejora de la calidad de vida y bienestar de los niños y niñas (Pineda y Orozco, 2018 p. 148).

De esta forma, el cumplimiento de dichos compromisos ha sido efecto de los diversos gobiernos colombianos, continuando con el esfuerzo por el posicionamiento de la primera infancia en los referentes de política pública en Colombia más allá de las posiciones políticas o los partidos políticos de Gobierno. En este sentido, se rescata el aumento presupuestal que de manera exponencial han tenido el Instituto de Bienestar Familiar ICBF, la Subdirección de Infancia y las oficinas de educación prescolar de los Ministerios de Educación y Cultura. En este sentido, los grupos de investigación $A B A$ de la Universidad Santo Tomas y Trabajo de llano de la Corporación Universitaria Minuto de Dios, ambas de la ciudad de Villavicencio en el departamento del Meta, han venido desarrollando el proyecto: Ecosistemas de Aprendizaje para la primera infancia con enfoque de ludificación, investigación que se enfoca por el diseño y la construcción de estrategias pedagógicas para la primera infancia bajo el enfoque de la ludificación; para tal fin, la investigación tiene población objeto niños y niñas entre los cinco y siete años de edad ubicados en la Ciudad de Villavicencio, Colombia., pertenecientes a colegios públicos y privados en asentamientos vulnerables y con bajos índices de necesidades básicas insatisfechas.

En este sentido, el proyecto en su primera y segunda fase se concentró en el desarrollo y aplicación de un diagnóstico inicial eficaz que identificara las capacidades y talentos naturales de los niños y niñas que asisten a hogares comunitarios, colegios y jardines infantiles públicos y privados en contextos vulnerables de cuatro asentamientos urbanos de la ciudad de Villavicencio (Pineda y Orozco, 2018, p. 148). El diagnostico se enfocó en cuatro variables previamente identificadas, el aprendizaje, la motivación, la conducta y el juego; este diagnóstico busco identificar situaciones de aprendizaje que posteriormente se pudieran potenciar para el óptimo desarrollo de la capacidad neuronal ya instalada en cada uno de los niños y niñas (Pineda et al, 2014). 
Paralelamente, al desarrollo del diagnóstico inicial, el grupo de investigación realizo un primer estado del arte de carácter exploratorio que pretendía documentar experiencias pedagógicas exitosas en el uso de la ludificación en el desarrollo de procesos de aprendizaje en la primera infancia; esto con el fin de poder establecer un modelo didáctico que fuera replicable en otras instituciones educativas que pretendan utilizar elementos de la ludificación como potencial educativo para la mejora de los aprendizajes de niños y niñas entre los cinco y siete años de edad.

Por tal razón, el presente artículo da cuenta de la segunda fase de exploración (2017 - 2018), la cual se concentró en una revisión exhaustiva de referentes teóricos, investigaciones y/o programas educativos que incluyeran elementos de ludificación en sus metodologías para desarrollo de aprendizajes en la primera infancia. En primer lugar, se presentará la problemática que da partida a la segunda fase de la investigación, posteriormente se identificaran los procesos de revisión documental de literatura especializada triangulada con los datos que arrojó el análisis hermenéutico de las prácticas y narrativas pedagógicas de los docentes involucrados, posteriormente se concluye recomendaciones propias para la implementación y abordaje de la ludificación en procesos educativos y pedagógicos con población en primera infancia. Claramente, este articulo complementa y amplia los hallazgos expuestos y publicados por Pineda y Orozco (2018), sobre la primea fase del análisis documental.

\section{Problemática}

Los avances de la investigación en neurociencia han logrado situar la discusión del aprendizaje humano nuevamente en boga. Las nuevas comprensiones sobre las formas de aprendizaje, los contextos y la durabilidad de estos, han permitido que el campo de la educación haya desempolvado sus antiguas discusiones sobre aprendizaje y didáctica y halla incorporado la compresión sobre el cerebro y sus funciones como tema recurrente en el diseño de currículos y estrategias de aprendizaje (Pineda y Orozco 2018b).

Las actuales tendencias sobre como sucede el proceso de aprendizaje han evidenciado nuevas comprensiones sobre el cerebro, su anatomía, sus funciones y como este influye notablemente sobre el comportamiento del sujeto, sobre sus maneras de interpretar representar, construir, compartir y apropiar la realidad y transformarla en conocimiento (Pineda y Orozco, 2018a, p. 149). 
Por tal razón, nos hemos planteado el interrogante sobre las didácticas del aula de clase y si ¿estas son coherentes con los hallazgos que desde las neurociencias y sus disciplinas subyacentes (neuroeducación, neuropsicología y neuropaprendizaje)? Para esto, es necesario evaluar no solo el conocimiento que los docentes tienen sobre dichos hallazgos sino la interpretación que se tiene de mitos basados en el funcionamiento del cerebro; asimismo, fue pertinente evaluar los conocimientos que docentes e instituciones educativas poseen sobre diseño de modelos pedagógicos y estrategias de enseñanza. Lo anterior, con el fin de nutrir la investigación sobre el diseño y construcción de un ecosistema de aprendizaje que contemple implicar nuevas fuentes de generación de nuevo conocimiento para poder traducirlas en metodologías que contribuyan a tener una educación de calidad para la primera infancia con prácticas pedagógicas actuales. (Pineda y Orozco, 2018c)

En la educación la manera como se concibe el proceso de aprendizaje está íntimamente ligada a la forma como se desarrolla la práctica pedagógica en la cotidianidad. Esta experiencia praxeológica mostró que los niños y niñas que comparten y conviven en espacios de aprendizaje donde el juego es un paradigma consistente y organizado acorde y coherente con sus potencialidades y necesidades de orden biológico, psicológico y afectivo generan mayores niveles de satisfacción y logro de aprendizaje (Pineda y Orozco, 2018a, p. 150)

A partir de lo anterior, la presente investigación busca evaluar la posibilidad de establecer un nuevo paradigma educativo para la primera infancia, donde la neuroeducación, la neuropsicología y la ludificación sean los componentes de los programas de atención y educación y ciudadano. Para tal fin, se diseñó una primera fase de revisión del estado del arte sobre ecosistemas de aprendizaje y ludificación (Pineda y Orozco, 2018a) y una complementaria revisión conceptual del uso de estrategias de ludificación y su relación con la práctica pedagógica en primera infancia y de esta forma poder establecer los parámetros básicos que podría tener un modelo educativo ludificado para la primera infancia

\section{La relación entre ludificación y primera infancia}

Con base en identificar la relación existente entre primera infancia y la ludificación, los procesos metodológicos de búsqueda y revisión documental siguen sin arrojar un estudio y/o investigación específica que incluya un modelo pedagógico y una estrategia de 
formación docente desde la ludificación o por lo menos, que incluyera elementos de esta en su proceso metodológico; por tal razón, se citaran estudios, investigaciones y acercamientos que desde otras áreas y disciplinas puedan abarcar lo enunciado en la problemática.

En este sentido, sigue siendo recurrente el tema de las dificultades del aprendizaje en el contexto de la primera infancia; allí se ubican investigaciones donde se enuncia la lúdica y el juego de forma explícita o implícita como elemento constitutivo para el abordaje de problemáticas y factores asociados a las dificultades de aprendizaje. De esta forma, se puede evidenciar como el juego y la lúdica posibilitan la integración de niños y niñas con diagnóstico de dificultades cognitivas y problemas de aprendizaje en un aula regular, el uso del juego educativo para el afianzamiento de procesos cognitivos y la utilidad del juego de roles para la formación ciudadana y la ética y moral en niños y niñas.

En este orden de ideas, se confirman los cuatros variables dependientes del estudio: la motivación, el juego, la conducta y el aprendizaje como fines del uso de estrategias lúdicas. En estos documentos analizados se evidencia una emergencia por deconstruir el significado de aprender a través del replanteamiento de estilos de aprendizaje y estilos de enseñanza centrados en procesos lúdicos. El análisis de los resultados publicados, permiten identificar tendencias conceptuales y metodológicas e identificar grupos de maestros activos interesados en trabajar en la línea del aprendizaje y sus dificultades desde sus propias narrativas y experiencias de aula.

Por otro lado, se evidencian estudios encaminados hacia la evaluación de aprendizajes en la primera infancia, evaluación que va de la mano de procesos disruptivos que docentes pretenden establecer para comprender procesos cognoscitivos en estudiantes de ciclos iniciales. En este sentido cabe resaltar, el instrumento de evaluación que combina la evaluación neuropsicológica de funciones ejecutivas (Blanco, Zea, Espitia y Acuña, 2013) junto con las estrategias de ludoestaciones relatas por Acuña (2008), consolidando de esta manera un instrumento y una estrategia de evaluación cognoscitiva en el desarrollo del pensamiento, aprendizaje y sus dificultades desde las perspectivas preventiva y de intervención pedagógica.

El complemento a dicho instrumento de evaluación desde la ludificación realizado por Blanco, Zea, Espitia y Acuña (2013), se encentar el proceso de formación docente desde los referentes de ludificación y neuroaprendizaje que Pineda, Paz y Cuervo (2014) 
aplican con más de 300 docentes de primera infancia en Colombia. Por otra parte, se destacan los quince proyectos de investigación desarrollados por estudiantes del programa de pedagogía infantil de la Corporación Universitaria Minuto de Dios UNIMINUTO en Villavicencio, los cuales relatan de manera clara los pormenores del uso de estrategias ludificadas para el aprendizaje de la lectura, la escritura y los saberes pre matemáticos, la formación ciudadana y la educación física y estética en 35 jardines y hogares infantiles en la ciudad de Villavicencio, Colombia.

Con lo anterior, se corrobora la hipótesis sobre la relación directa entre ludificación y primera infancia ya sea desde el uso de la lúdica como estrategia pedagógica o como referente para la evaluación de aprendizajes a través del juego o como didáctica específica para el óptimo y eficaz desarrollo de procesos cognoscitivos, emocionales y comportamentales en niños y niñas escolarizados. Con esto, la investigación se propuso realizar el estado del arte sobre dicha relación y de esta forma, poder evaluar, conocer y comprender diferentes experiencias que vinculen la ludificación y la primera infancia desde las variables de conducta, motivación, juego y aprendizaje.

\section{Metodología}

Para el desarrollo de esta fase, se adopta la investigación cualitativa- y se aplica la estrategia investigativa que propone Pineda y Velásquez (2015) desde la sistematización de experiencias a partir de la perspectiva del enfoque praxeologico. Para tal fin, se establecen cuatro criterios de desarrollo de la investigación (I) Ver, (II) Juzgar, (III) Actuar y (IV) Devolución Creativa. En este sentido, en la investigación desde el enfoque praxeológico se tienen en cuenta fundamentalmente cuatro fases:

a. Se busca la participación de la comunidad investigada y que tiene problemas por solucionar a lo largo de todo el proceso de investigación. b. Se investiga sobre su propia realidad, en ella los sujetos de la investigación son los protagonistas de su propio proceso investigativo. c. Se establece una nueva relación teoría práctica (praxis). d. Se determinan las necesidades y se formula una estrategia creativa para dar solución a la problemática identificada (Pineda y Orozco, 208, p. 153).

De esta forma, se evidencia como el proceso investigativo está comprometido ética y políticamente con la trasformación de los modelos pedagógicos para la primera infancia y se fundamenta en el abordaje pedagógico de la atención a la primera infancia. Entonces, en una segunda etapa se centra el abordaje teórico de los procesos 
cognoscitivos como una mirada hacia los dispositivos básicos de aprendizaje, identificando aspectos relevantes y conceptos que determinan el trabajo pedagógico en la primera infancia (Orozco y Pineda, 2017).

De esta manera, según los criterios expuestos por Pineda y Velásquez (2015) se plantearon para el proceso de la revisión documental de la relación entre el concepto de ludificación y primera infancia abordar la fase 2 correspondiente a la estrategia del $J U Z G A R$

Fase 2 - JUZGAR

Fase desarrollada durante los años 2016 y 2017 tuvo como objetivo realizar una revisión documental de literatura especializada en Ludificación, Teoría del Juego, Gamificación, Neuroaprendizaje, Ludoestaciones; así como de realizar un análisis comparativo de los modelos pedagógicos de catorce colegios y veinticinco jardines infantiles de la ciudad de Villavicencio, Colombia perteneciente al proyecto. En esta misma fase se desarrolló el estado del arte que sobre ludificación aplicada a la educación en primera infancia se encontró en revisión de bases de datos especializadas.

\section{Resultados}

\section{Estado del arte de la relación entre ludificación y primera infancia}

Para Zichermann y Cunningham (2011) la ludificación se afronta como "un proceso relacionado con el pensamiento del jugador y las técnicas de juego para atraer a los usuarios y resolver problemas" (Zichermann y Cunningham, 2011, p.11). Así mismo, para Kapp (2012), la ludificación se toma como "la utilización de mecanismos, la estética y el uso del pensamiento, para atraer a las personas, incitar a la acción, promover el aprendizaje y resolver problemas" (Kapp, 2012, p.9). Es de resaltar como los tres autores argumentan que la finalidad de todo juego es influir en la conducta psicológica y social del jugador: por tal razón indican que a través del uso de ciertos elementos presentes en los juegos (emblemas, puntos, distintivos, niveles, avatar, etc.) los jugadores incrementan su tiempo en el juego, así como su predisposición psicológica a seguir en él.

En la revisión documental sobre ludificación se evidenciaron autores que hacen una distinción entre la ludificación y los videojuegos, entre ellos se encuentra Hamari y Koivisto (2014), quienes establecen las diferencias entre juegos ludificados y videojuegos: (i) La ludificación busca intervenir en el comportamiento de las personas. 
(ii) La ludificación se basa en la experiencia vinculada a las emociones y a los cambios de comportamiento que estos acaecen. (iii) Los videojuegos están basados exclusivamente en una satisfacción a través de la narrativa audiovisual. En ese mismo sentido, Kapp (2012) relata la diferencia que existe entre la gamificación y los juegos educativos en las aulas, mostrando que la ludificación presenta un espacio lúdico más atractivo para los estudiantes mientras que los juegos educativos no.

Se evidencio como en los autores Kapp (2012) y Zichermann y Cunningham (2011) es reiterativa la enunciación de los elementos del juego en referencia a las acciones acaecidas por el mismo ser humano. Se puede inferir en estos atores como la ludificación es un ejercicio de reflexión sobre las prácticas y no es ajena a la estructura investigativa que propone Juliao (2011) en el enfoque praxeológico, determinando ambos las diferentes actitudes que poseen los sujetos para conseguir un determinado bien (ganancias) y es así como, para que las personas tengan una conducta concreta guiada por la auto reflexión y el constante mejoramiento de las practicas se crea un sistema adecuado que logra obtener el comportamiento deseado.

Dentro de los documentos analizados de Kapp (2012), Zichermann y Cunningham (2011), sobresale la citación de conceptos relacionados con la adquisición de logros, entendido este elemento como constitutivo del juego (logro) y objeto de estudios en otras áreas y disciplinas (Vassileva, 2012). Otro de los elementos del juego con mayor citación en los autores es el reto, este concepto se aborda desde una significante carga psicológica y cuyo enfoque es la influencia en el comportamiento del sujeto (usuario - jugador). Continuando con el análisis documental se encuentran autores como Tejeiro y Pelegrina (2008), los cuales realizan una aproximación a la relación entre la psicología y los videojuegos, haciendo énfasis en los sujetos usuarios, quienes son conscientes de las exigencias del juego, de sus limitantes y posibilidades, siendo los primeros en anotar e interiorizar el reto como motivante para el abordaje de un videojuego (Tejeiro y Pelegrina, 2008).

Por otra parte, se encuentra Shotton (1992) el cual sugiere que los videojuegos además de colaborar en el incremento de la destreza motriz y la coordinación viso-motora, permitiendo un incremento en las sinapsis, permitiendo que el conocimiento adquirido incremente su velocidad neuronal acelerando de esta manera juicios, toma de decisiones y resolución de problemas. Así mismo Johnson (2012) demanda como las diferentes 
mediaciones e hipermediaciones (Scolari, 2010) que influyen en los sujetos depende de las necesidades y condicionamientos impuestos por la misma sociedad; es decir los videojuegos de hoy en día se estructuran en retos y logros acordes a las capacidades de los sujetos contemporáneos.

En el ámbito educativo, la revisión documental se centró en los postulados de Lee, Ceyhan, Jordan-Cooley y Sung (2013), los cuales afirman que la ludificación aplicada al contexto educativo puede convertirse en un sistema práctico que ofrecería resoluciones agiles con las que los estudiantes logren desarrollar aprendizajes constantes mediados por experiencias gratificantes. Además, como sistema educativo, puede resultar atractivo teniendo en cuenta que "la gamificación puede ser una estrategia de gran alcance que promueva la educación entre las personas y un cambio de comportamiento" (Ceyhan, Jordan-Cooley y Sung (2013, p.15); por lo tanto, la ludificación en el espacio educativo y académico puede crear un cambio en la actitud de los estudiantes y profesores apartado de prácticas de coerción y engaño, utilizando para eso elementos del juego y de los videojuegos que logren captar la atención de los estudiantes generando por si un cambio de actitud en el comportamiento de los mismos (Ermi y Mäyrä, 2005).

Por otra parte, Hamari (2014), realizan un análisis de las diferentes publicaciones de investigaciones sobre ludificación, haciendo un análisis hermenéutico sobre la definición del concepto de ludificación, los elementos del juego en contexto, el contexto ludificado y los usuarios del mismo; como conclusión Hamari (2014) enfatiza en la presencia de deficiencias metodológicas referentes a tamaños de muestra pequeños, falta de grupos control, sesgos de interpretación en cuanto el concepto de ludificación, tiempos de investigación demasiados cortos y falta de claridad en el análisis de los resultados a la luz de la ludificación.

Para Hamari (2014), la ludificación viene en auge en la literatura de corte académico e investigativo, ya sea por la vinculación del término en el titulo de los artículos como en su enunciamiento en los marcos teóricos de los mismos. Hamari (2014) llaman la atención sobre si se usa coherentemente el termino o si por el contrario se adaptan ejercicios de la lúdica, teoría del juego a la ludificación. Para Hamari et al (2014) la ludificación se puede abordar desde tres estadios (Ver figura 1) 
Figura 1. Estadios de la ludificación

\begin{tabular}{|l|l|l|}
\hline $\begin{array}{l}\text { Posibilidades de acción } \\
\text { motivacional }\end{array}$ & Resultados Psicológicos & Resultados Conductuales \\
\hline
\end{tabular}

Fuente(s): Hamari, 2014

Los diferentes autores analizados permiten inferir las necesidades metodológicas que desde el campo educativo y del aprendizaje se deben tener en cuenta para la coherente definición aplicada del término de gamificación. Si bien, la gamificación o ludificación ha tenido relativo éxito en áreas del marketing y la economía y las relaciones humanas, es muy incipiente el record de experiencias que desde la academia se organicen para la inclusión de la gamificación en los procesos de aprendizaje y más aún lo correspondiente a gamificación y aprendizaje en la infancia.

\section{Revisión documental}

En primera medida, para poder establecer un parámetro de búsqueda se realizó la revisión documental en bases de datos generales; para tal efecto, se utilizó el navegador Google, digitando primero la palabra ludificación obteniendo 54200 resultados, seguidamente se buscó la palabra gamificación obteniendo 94500 resultados, estos indicen evidencian un alto número de hallazgos en bases de datos, páginas web y repositorios de información. Seguido se anexó a cada búsqueda la palabra artículos obteniendo que para la combinación ludificación-artículos se encontraron 29.100 resultados y para la combinación gamificación-artículos se encontraron 107.000 resultados. En la plataforma Google Scholar se obtuvieron 87 resultados para la palabra gamificación, 13 resultados para ludificación.

Seguido, se aplicó validez de resultados en la primera y segunda búsqueda a través del ingreso a cuatro resultados de cada una de las páginas cargadas en Google y Google Scholar, teniendo como resultado que el $64 \%$ de las páginas a las que se accedieron son del campo de las TIC; el 23\% son páginas de marketing y comercio, el 11\% páginas referentes a blogs y wikis y solo un $2 \%$ pertenecientes a páginas académicas de universidades y/o revistas científicas.

Posteriormente, se realizaron búsquedas en las siguientes bases de datos: Scopus, EBSCOHost, SciElo, Dialnet, Proquest; los términos de búsqueda fueron los mismos que se utilizaron en Google, los términos de búsqueda se utilizaron para todos los campos 
(título, resumen, palabras clave y texto completo), y para todos los tipos de resultados (Tabla 1).

Tabla 1. Resultados por base de datos.

\begin{tabular}{|c|c|c|c|c|}
\hline \multirow{2}{*}{ Base de Datos } & \multicolumn{3}{|c|}{ Total de resultados } & \multirow{2}{*}{$\begin{array}{l}\text { No documentos } \\
\text { revisados }^{1}\end{array}$} \\
\hline & Gamificación & Ludificación & gamification $^{2}$ & \\
\hline EBSCO & 4 & 5 & 1630 & 8 \\
\hline Proquest & 6 & 1 & 199 & 7 \\
\hline Scopus & 5 & 0 & 864 & 5 \\
\hline Google $^{3}$ & 94500 & 54200 & 6900000 & 0 \\
\hline $\begin{array}{l}\text { Google } \\
\text { Scholar }\end{array}$ & 87 & 13 & 14500 & 0 \\
\hline Dialnet & 51 & 6 & 24 & 36 \\
\hline
\end{tabular}

Fuente(s): autores.

Las búsquedas focalizadas fueron realizadas con los siguientes criterios previos:

- Documento con revisión por pares publicado en un instancia nacional o internacional reconocida.

- Perteneciente a un estudio y/o investigación y que su metodología y resultados estuviera incluido en el texto.

- Se especifique el método de investigación utilizado.

- Se comprenda claramente la motivación o problemática abordada.

- Que el estudio nombrara la gamificación o ludificación como objeto de estudio y/o implementación y no se equiparara a la teoría de juegos y/o a la lúdica.

Con los anteriores criterios, se condicionó el análisis de documentos y se limitó la búsqueda. Se analizaron aquellos documentos seleccionados para la muestra y que no cumplían los requisitos previos y se establecieron las siguientes categorías:

1. Son documentos conceptuales que no responden a resultados $y / 0$ análisis de estudios y/o investigaciones.

\footnotetext{
${ }^{1}$ Los documentos revisados fueron seleccionados a través de fórmula matemática para identificar el $n$ de cada base de datos. A su vez se verifico que los documentos seleccionados hayan sido sometidos a revisión por pares y contengan la rigurosidad pertinente.

${ }^{2}$ Se realizó la búsqueda de la palabra anglosajona Gamification solo para generar comparación entre documentos escritos en inglés y los escritos en español. para el trabajo se analizaron solo documentos escritos en español.

${ }^{3}$ De las plataformas Google y Google Scholar no se revisaron documentos sino validación de páginas.
} 
2. Son documentos del campo de la ingeniería que describen un sistema y carecen de evaluación por pares.

3. No se define ni se conceptualizan los términos de gamificación o ludificación en el texto.

4. Se enuncia en el texto los conceptos de gamificación o ludificación, pero no constituye el corpus de la disertación y/o discusión.

5. Tipología de documentos breves / investigación en curso / resúmenes

Después de realizar los filtros correspondientes se establecieron para el análisis y revisión veinticinco (25) trabajos de investigación revisados por pares en el tema de Gamificación y/o ludificación.

Tabla 2. Matriz recolección información.

\begin{tabular}{|l|ll|}
\hline Revisión Bibliográfica & \\
\hline Concepto - Ubicación & Autor - Ubicación & \\
\hline Concepto Ludificación (Autor a, Autor b, etc.) & $\begin{array}{l}\text { Autor a. (Concepto ludificación y/o } \\
\text { gamificación) }\end{array}$ \\
\hline
\end{tabular}

Fuente(s): Webster y Watson, 2002

Tabla 3. Matriz conceptos.

\begin{tabular}{|l|l|l|}
\hline Matriz de Conceptos & \multicolumn{2}{|l|}{} \\
\hline \multirow{2}{*}{ Artículos } & Conceptos & Ludificación \\
\cline { 2 - 3 } & Gamificación & \\
\hline 1 & $\mathrm{x}$ & $\mathrm{x}$ \\
\hline 2 & $\mathrm{x}$ & $\mathrm{x}$ \\
\hline 3 & & \multicolumn{2}{|l|}{} \\
\hline
\end{tabular}

Fuente(s): Webster y Watson, 2002

Tabla 4. Matriz según unidades de análisis motivacional

\begin{tabular}{|c|c|c|c|c|c|c|c|c|}
\hline \multicolumn{9}{|c|}{ Matriz según unidades de análisis motivacional } \\
\hline Articul & \multicolumn{8}{|c|}{ Análisis motivacional TIC } \\
\hline $\begin{array}{l}\text { Unidad } \\
\text { de } \\
\text { análisis }\end{array}$ & $\begin{array}{l}\text { Autonomí } \\
\text { a }\end{array}$ & $\begin{array}{l}\text { Competenci } \\
\text { a }\end{array}$ & $\begin{array}{l}\text { Logr } \\
\text { o }\end{array}$ & $\begin{array}{l}\text { Ret } \\
\text { o }\end{array}$ & $\begin{array}{l}\text { Liderazg } \\
\mathrm{o}\end{array}$ & $\begin{array}{l}\text { Emoció } \\
\mathrm{n}\end{array}$ & $\begin{array}{l}\text { Motivació } \\
\mathrm{n}\end{array}$ & $\begin{array}{l}\text { Resilienci } \\
\text { a }\end{array}$ \\
\hline Alto & $\mathrm{x}$ & & $\mathrm{x}$ & $\mathrm{x}$ & $\mathrm{x}$ & & $\mathrm{X}$ & \\
\hline Medio & & $\mathrm{X}$ & & & & $\mathrm{x}$ & & \\
\hline Bajo & & & & & & & & $\mathrm{x}$ \\
\hline Nulo & & & & & & & & \\
\hline
\end{tabular}

Fuente(s): autores. 
Tabla 5. Registro Bibliográfico

\begin{tabular}{|l|l|l|l|}
\hline Registro & Titulo & \\
\hline Fuente & & Ubicación & \\
\hline Tipo de Documento & & Palabras Clave & \\
\hline Tema & & \\
\hline Resumen Abstract & & \\
\hline Observaciones & & \\
\hline
\end{tabular}

Fuente(s): autores.

\section{Análisis}

Para el análisis de los documentos y la presentación de resultados, se combinó un modelo de motivación hacia las TIC que contemplara necesidades, cogniciones y emociones con una versión modificada de la matriz de Webster y Watson (2002) para tener una matriz sistemática de recopilación y análisis de los diferentes documentos (tabla 2,3,4). Se analizaron los documentos y se clasificaron por los conceptos previstos de gamificación o ludificación. Los resultados del análisis se reunieron en dos tablas, Una de registro (tabla 5) y otra de análisis (Tabla 6).

Tabla 6. Tabla de análisis documental

\begin{tabular}{|l|l|}
\hline Análisis Documental & \\
\hline Titulo & \\
\hline Autor (es) & \\
\hline Institución & \\
\hline Fuente & \\
\hline Ubicación & \\
\hline Año & \\
\hline Autor & \\
\hline Ciudad / País & \\
\hline Palabras Clave & \\
\hline Resumen & \\
\hline Introducción & \\
\hline Objetivos & \\
\hline Marco de referencia & \\
\hline Tipo de Investigación & \\
\hline Población & \\
\hline Muestra & \\
\hline Contexto & \\
\hline Instrumentos & \\
\hline Metodología & \\
\hline Resultados & \\
\hline Análisis & \\
\hline Conclusiones & \\
\hline Recomendaciones & \\
\hline Bibliografía & \\
\hline Fecha de Elaboración & \\
\hline
\end{tabular}

Fuente(s): autores. 
Tabla 7. Matriz información artículos

\begin{tabular}{|c|c|}
\hline Revisión Bibliográfica & \\
\hline Concepto - Ludificación - Gamificación & \\
\hline Documento & Autor \\
\hline $\begin{array}{l}\text { (1) Analizando y aplicando la gamificación en } \\
\text { el proceso ChildProgramming }\end{array}$ & Orejuela, García, Hurtado y Collazos (2013) \\
\hline $\begin{array}{l}\text { (2) Aspectos básicos de la gamificación en } \\
\text { Educación Infantil }\end{array}$ & Ciganda Azkárate, I (2018) \\
\hline $\begin{array}{l}\text { (3) La gamificación en el aprendizaje de } \\
\text { lenguas extranjeras en Educación Primaria: } \\
\text { revisión de estudios y propuestas }\end{array}$ & Mangado Aguirre, P. (2016) \\
\hline $\begin{array}{l}\text { (4) Didáctica, semiótica y gamificación } \\
\text { matemática no digital en niños de un complejo } \\
\text { municipal asistencial infantil }\end{array}$ & $\begin{array}{l}\text { Holguín Álvarez, J.A.; Villa Córdova, G.M.; } \\
\text { Baldeon de la Cruz, M. y Chávez Álvarez, Y. } \\
\text { (2017) }\end{array}$ \\
\hline $\begin{array}{l}\text { (5) Gamificación en educación primara. Un } \\
\text { estudio piloto desde la perspectiva de sus } \\
\text { protagonistas }\end{array}$ & Carrión Salinas, G.A. (2017) \\
\hline $\begin{array}{l}\text { (6) La utilización de la gamificación para la } \\
\text { mejora de la educación en alumnado con } \\
\text { Trastorno de Déficit de Atención e } \\
\text { Hiperactividad }\end{array}$ & Mudarra Peña, E. (2015) \\
\hline $\begin{array}{l}\text { (7) La gamificación educativa. Una alternativa } \\
\text { para la enseñanza creativa }\end{array}$ & Marín Díaz, V. (2015) \\
\hline $\begin{array}{l}\text { (8) El uso de herramientas } 2.0 \text { como recursos } \\
\text { innovadores en el aprendizaje de niños y niñas } \\
\text { en Educación Infantil. Un estudio de caso de } \\
\text { investigación-acción }\end{array}$ & $\begin{array}{l}\text { Gertudrix Barrio, F.; Ballesteros Ávila, V. } \\
\text { (2014) }\end{array}$ \\
\hline $\begin{array}{l}\text { (9) De la enseñanza con libros de texto al } \\
\text { aprendizaje en espacios online gamificados }\end{array}$ & Moreira M.A.; González, C.S.G. (2015) \\
\hline $\begin{array}{l}\text { (10) Interconectados apostando por la } \\
\text { construcción colectiva del conocimiento. } \\
\text { Aprendizaje móvil en educación infantil y } \\
\text { primaria }\end{array}$ & Quintana J.G. (2017) \\
\hline $\begin{array}{l}\text { (11) Evaluación y rediseño de una experiencia } \\
\text { de gamificación en el aula basada en estilos de } \\
\text { aprendizaje y tipos de jugador }\end{array}$ & Baldeón, J.; Rodríguez, I. Puig, A. (2017) \\
\hline $\begin{array}{l}\text { (12) El dibujo de la figura humana "Avatar" } \\
\text { como elemento para el desarrollo de la } \\
\text { creatividad y aprendizaje a través de } \\
\text { la gamificación en Educación Primaria }\end{array}$ & Gaitero, FG. Domínguez, SC (2016) \\
\hline $\begin{array}{l}\text { (13) La gamificación con scratch como rincón } \\
\text { de aprendizaje para el subnivel dos del } \\
\text { currículo de educación inicial de los alumnos } \\
\text { de educación inicial del centro de desarrollo } \\
\text { infantil "Caritas Felices" durante el periodo } \\
\text { lectivo } 2015 \text { - 2016. Lineamientos propositivos }\end{array}$ & Charchabal, D. Veja Trujillo, P.A. (2016) \\
\hline $\begin{array}{l}\text { (14) Aspectos básicos de la gamificación en } \\
\text { Educación Infantil }\end{array}$ & Ciganda Azkárate, I. (2017) \\
\hline $\begin{array}{l}\text { (15) Gamificación: Estrategia para optimizar el } \\
\text { proceso de aprendizaje y la adquisición de } \\
\text { competencias en contextos universitarios }\end{array}$ & $\begin{array}{l}\text { Villalustre Martínez, L. , } \\
\text { Del Moral Pérez, M.E. }\end{array}$ \\
\hline $\begin{array}{l}\text { (16) La gamificación como estrategia de } \\
\text { motivación en Educación Infantil }\end{array}$ & Barragán Moreno, AB (2017) \\
\hline (17) Y para aprender, el cerebro se puso a jugar & Marín, I. Forés, A. Hierro Mariné, E. \\
\hline (18) Videojuegos y aprendizaje & Martín del Pozo, M. \\
\hline $\begin{array}{l}\text { (19) Intervención multidisciplinar en afasia } \\
\text { desde el modelo neuropsicológico }\end{array}$ & García López, C. Marín Suelves, D. \\
\hline
\end{tabular}




\begin{tabular}{|l|l|}
\hline $\begin{array}{l}\text { (20) La Gamificación al servicio de nuevos } \\
\text { modelos de comunicación surgidos de la } \\
\text { cibercultura }\end{array}$ & Vargas-Machuca, R. Rosano, F. \\
\hline $\begin{array}{l}\text { (21) El papel de los videojuegos en el } \\
\text { desarrollo cognitivo }\end{array}$ & Carvajal Garrido, D. \\
\hline $\begin{array}{l}\text { (22) Una experiencia de gamificación con } \\
\text { tablets para potenciar el inglés en un aula de } \\
\text { infantil }\end{array}$ & Gallardo Ibáñez, P (2017) \\
\hline $\begin{array}{l}\text { (23) La gamificación en el aula de 1o. de de } \\
\text { Educación Infantil: Una propuesta matematiza- } \\
\text { geométrica }\end{array}$ & Cuadrado Antolín, A. (2017) \\
\hline (24) BN y Gamificación en Educación Infantil & Ramos García, C. (2017) \\
\hline $\begin{array}{l}\text { (25) Ecosistemas de aprendizaje bilingües: una } \\
\text { experiencia de trasferencia pedagógica desde la } \\
\text { gamificación }\end{array}$ & Orozco, P. Pineda, E. (2017) \\
\hline
\end{tabular}

Fuente(s): autores.

\section{Resultados}

En total se examinaron 25 documentos. En todos los documentos se encontraron elementos conceptuales suficientes que den respuesta a la pregunta de investigación referida a si ¿funciona o no la gamificación o ludificación?, se encontraron coincidencias sobre la pertinencia de la gamificación o ludificación en los espacios a implementar. Se trabaja más el concepto de gamificación que de ludificación en los documentos analizados.

Como factores motivacionales se encuentra presente con mayor ahincó los referentes al logro y al reto (90\% de documentos); competencia y autonomía con presencia frecuente $(75 \%)$ y con alta presencia $(60 \%)$ emoción, motivación, resiliencia y aprendizaje. las variaciones entre gamificación (español) y gamification (inglés) son frecuentes en los documentos analizados en un porcentaje del 74\%. En todos los documentos analizados se enuncian elementos del juego a ser aplicados en contextos no ludificados.

Tabla 8. Matriz conceptos

\begin{tabular}{|l|l|l|}
\hline \multicolumn{2}{|l|}{ Matriz de Conceptos } \\
\hline \multirow{2}{*}{ Artículos } & Conceptos & \\
\cline { 2 - 3 } & Gamificación & Ludificación \\
\hline 1 & $\mathrm{X}$ & $\mathrm{X}$ \\
\hline 2 & $\mathrm{X}$ & \\
\hline 3 & $\mathrm{X}$ & $\mathrm{X}$ \\
\hline
\end{tabular}




\begin{tabular}{|c|c|c|}
\hline 4 & $X$ & \\
\hline 5 & $X$ & \\
\hline 6 & $X$ & $X$ \\
\hline 7 & $X$ & \\
\hline 8 & $X$ & $X$ \\
\hline 9 & $X$ & \\
\hline 10 & $X$ & $X$ \\
\hline 11 & $X$ & $X$ \\
\hline 12 & $X$ & \\
\hline 13 & $X$ & \\
\hline 14 & $X$ & \\
\hline 15 & $X$ & \\
\hline 16 & $X$ & \\
\hline 17 & $X$ & \\
\hline 18 & $X$ & \\
\hline 19 & $X$ & \\
\hline 20 & $X$ & \\
\hline 21 & $X$ & \\
\hline 22 & $X$ & \\
\hline 23 & $X$ & \\
\hline 24 & $X$ & \\
\hline 25 & $X$ & \\
\hline 26 & $X$ & \\
\hline 27 & $X$ & \\
\hline
\end{tabular}

Fuente(s): autores.

Se presenta un alto porcentaje de aplicación y citación de conceptos de gamificación y ludificación en contextos de aprendizaje infantil (90\%), en entornos universitarios se presenta un $1 \%$, en entornos culturales $5 \%$ y un $1 \%$ en entornos clínicos 
y terapéuticos (psicología), un 3\% en estudios y/o investigaciones en contextos de educación media.

La mayoría de los documentos examinados $(90 \%)$ abordan procesos conductuales, enfocándose en experimentos o análisis estadísticos de implementaciones diseñadas por los investigadores desde un entorno de gamificación o ludificación en la búsqueda de un cambio comportamental y/o conductual. Se presenta un alto porcentaje (95\%) de reportes significativos hacia una intencionalidad de aprendizajes y procesos cognitivos a desarrollar a través de la aplicación y/o implementación de proceso de gamificación o ludificación.

De acuerdo con la totalidad de los documentos revisados, se asegura que la gamificación o ludificación sí produce efectos positivos y beneficiosos para los sujetos de investigación. En la tabla 8 se identifica que, la mayoría de los trabajos revisados presentan resultados positivos en el campo de lo motivacional. En cuanto a la metodología de investigación que presentan la mayoría de documentos, esta responde a trabajos cualimetricos que cuentan con procesos analíticos y descriptivos con solida metodología y método de aplicación, este proceso descriptivo relata implementaciones positivas sin embargo no se presentan comparaciones pre y post aplicación de la estrategia de gamificación o ludificación. EL 90\% de los documentos presentan una descripción clara de la población y de la muestra aplicada. No se presentan estudios a largo plazo (más de tres años) y si la mayoría presenta aplicaciones cortas en el tiempo sin análisis de efectos post-aplicación. No se presentan datos de carácter negativo en la implantación de la gamificación o ludificación

Tabla 8. Matriz conceptos

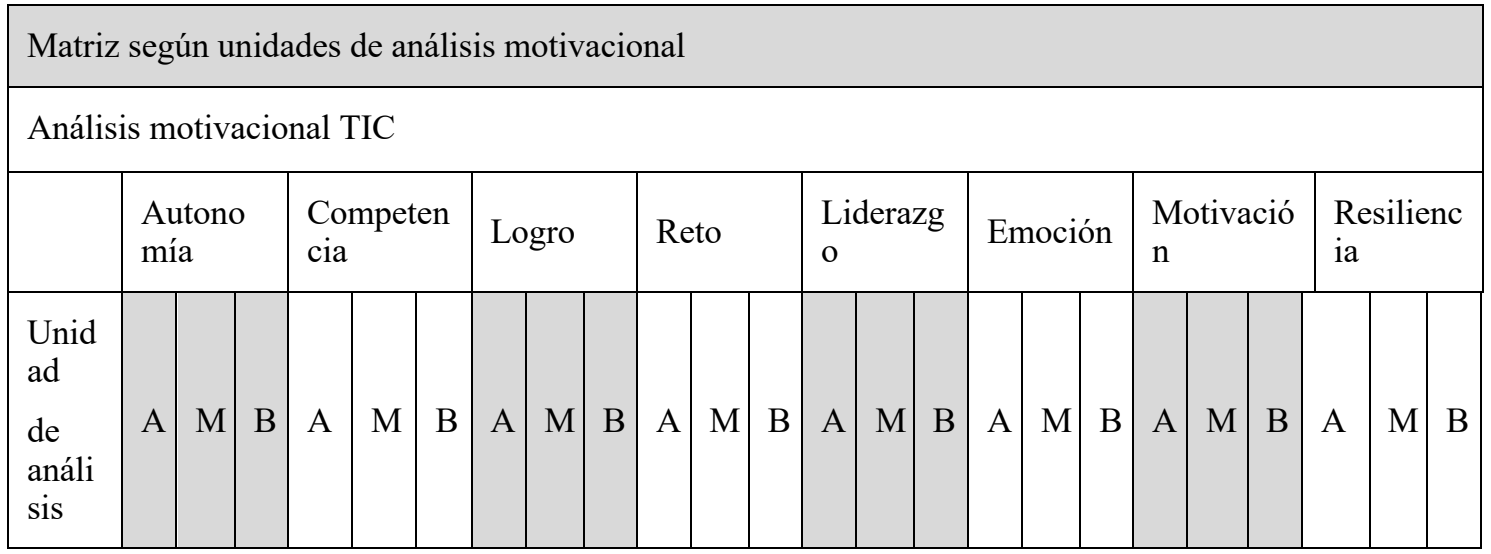




\begin{tabular}{|c|c|c|c|c|c|c|c|c|c|c|c|c|c|c|c|c|c|c|}
\hline $\begin{array}{l}\text { Art } \\
\text { ulo }\end{array}$ & & & & & & & & & & & & & & & & & & \\
\hline 1 & $\mathrm{X}$ & & $\mathrm{X}$ & & & $\mathrm{X}$ & & $x$ & & & $\mathrm{X}$ & & $\mathrm{X}$ & & $\mathrm{X}$ & & $\mathrm{X}$ & \\
\hline 2 & $\mathrm{X}$ & & $\mathrm{X}$ & & $X$ & & $X$ & & $\mathrm{X}$ & & & $\mathrm{X}$ & & $\mathrm{X}$ & & & $\mathrm{X}$ & \\
\hline 3 & $\mathrm{X}$ & & $\mathrm{X}$ & & & $x$ & & $x$ & & $\mathrm{X}$ & & & $\mathrm{X}$ & $\mathrm{x}$ & & & $\mathrm{X}$ & \\
\hline 4 & $\mathrm{X}$ & & $X$ & & & $\mathrm{X}$ & & $x$ & & $\mathrm{X}$ & & & $\mathrm{X}$ & & $X$ & & $\mathrm{X}$ & \\
\hline 5 & $\mathrm{X}$ & & $X$ & & & $\mathrm{X}$ & & $X$ & & & $\mathrm{X}$ & & $\mathrm{X}$ & . & $X$ & & $\mathrm{X}$ & \\
\hline 6 & $\mathrm{X}$ & & $X$ & & $\mathrm{X}$ & 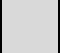 & $X$ & & $X$ & & & $\mathrm{X}$ & & $\mathrm{x}$ & & & $\mathrm{X}$ & \\
\hline 7 & & $\mathrm{X}$ & $X$ & & & $\mathrm{X}$ & $X$ & & $\mathrm{X}$ & & & & $\mathrm{X}$ & $\mathrm{X}$ & & & & $X$ \\
\hline 8 & & $\mathrm{X}$ & $X$ & & & $x$ & $X$ & & $\mathrm{X}$ & & & $\mathrm{X}$ & & $\mathrm{x}$ & & $\mathrm{X}$ & & \\
\hline 9 & $\mathrm{X}$ & & & $X$ & & $\mathrm{X}$ & & $x$ & $\mathrm{X}$ & 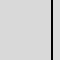 & & $\mathrm{X}$ & & $\mathrm{X}$ & & & & $X$ \\
\hline 10 & & $\mathrm{X}$ & $X$ & & & $x$ & $\mathrm{X}$ & & $\mathrm{X}$ & & & $\mathrm{X}$ & & $\mathrm{x}$ & & & $\mathrm{X}$ & \\
\hline 11 & $\mathrm{X}$ & & $X$ & & $\mathrm{X}$ & . & $\mathrm{X}$ & & $\mathrm{X}$ & & & $\mathrm{X}$ & & $\mathrm{X}$ & & $\mathrm{X}$ & & \\
\hline 12 & & $\mathrm{X}$ & & $X$ & & $\mathrm{X}$ & & $X$ & . & $\mathrm{X}$ & & & $\mathrm{X}$ & $\mathrm{X}$ & & & $\mathrm{X}$ & \\
\hline 13 & $\mathrm{X}$ & & $x$ & & $\mathrm{X}$ & & $X$ & & $X$ & & & $\mathrm{X}$ & & $X$ & & $X$ & & \\
\hline 14 & & $\mathrm{X}$ & & $X$ & & $\mathrm{X}$ & & $x$ & $\mathrm{X}$ & . & & & $\mathrm{X}$ & $\mathrm{x}$ & & & $\mathrm{X}$ & \\
\hline 15 & & $X$ & & $X$ & & $\mathrm{X}$ & $X$ & & $X$ & & & & $\mathrm{X}$ & $\mathrm{X}$ & & & $\mathrm{X}$ & \\
\hline 16 & & $\mathrm{X}$ & $\mathrm{X}$ & & $\mathrm{X}$ & & $\mathrm{X}$ & & $\mathrm{X}$ & & & & $\mathrm{X}$ & $\mathrm{X}$ & & & $\mathrm{X}$ & \\
\hline 17 & $\mathrm{X}$ & & $\mathrm{X}$ & & $\mathrm{X}$ & & $X$ & & $\mathrm{X}$ & & & $\mathrm{X}$ & & $\mathrm{X}$ & & & $\mathrm{X}$ & \\
\hline 18 & $\mathrm{X}$ & & $\mathrm{X}$ & & $\mathrm{X}$ & & $X$ & & $X$ & & & $\mathrm{X}$ & & $\mathrm{X}$ & & & $\mathrm{X}$ & \\
\hline 19 & $\mathrm{X}$ & & $\mathrm{X}$ & & $\mathrm{X}$ & & $X$ & & $\mathrm{X}$ & & & $\mathrm{X}$ & & $\mathrm{X}$ & & & $\mathrm{X}$ & \\
\hline 20 & & $\mathrm{X}$ & $\mathrm{X}$ & & $\mathrm{X}$ & & $X$ & & & $\mathrm{x}$ & & $\mathrm{X}$ & & $\mathrm{X}$ & & & $\mathrm{X}$ & \\
\hline 21 & $\mathrm{X}$ & & $\mathrm{X}$ & & $\mathrm{X}$ & & $\mathrm{X}$ & & $\mathrm{X}$ & & & & $\mathrm{X}$ & $\mathrm{X}$ & & & $\mathrm{X}$ & \\
\hline 22 & $\mathrm{X}$ & & $\mathrm{X}$ & & $\mathrm{X}$ & & $\mathrm{X}$ & & $X$ & & & & $\mathrm{X}$ & $\mathrm{X}$ & & & $\mathrm{X}$ & \\
\hline 23 & $\mathrm{X}$ & & $\mathrm{X}$ & & $\mathrm{X}$ & & $X$ & & $\mathrm{X}$ & & & $\mathrm{X}$ & & $\mathrm{X}$ & & & $\mathrm{X}$ & \\
\hline 24 & $\mathrm{X}$ & & & $X$ & $\mathrm{X}$ & & $X$ & & $X$ & & & & $\mathrm{X}$ & $\mathrm{x}$ & & & $\mathrm{X}$ & \\
\hline 25 & $\mathrm{X}$ & & & $X$ & $\mathrm{X}$ & & & $\mathrm{X}$ & $\mathrm{X}$ & & & & $\mathrm{X}$ & $\mathrm{X}$ & & & $\mathrm{x}$ & \\
\hline
\end{tabular}

$A=$ Adecuado $M=$ Medio $B=$ Bueno 


\section{Fuente(s): autores.}

Es de destacar la gama de contextos donde se da la formulación y se realizaron los estudios y/o investigaciones, presentando la educación o el aprendizaje como el contexto más común para las implementaciones, seguido por contextos de TIC, salud y deporte e innovación.

\section{Discusión}

En este trabajo se concentraron los esfuerzos en poder delimitar la relación entre gamificación o ludificación y primera infancia; por tal razón, la revisión documental realizada permite inferir la necesidad de conceptualizar la investigación a realizar con el fin de nutrir el campo disciplinar ante el auge en los últimos dos años de reportes en este campo especifico.

Se debe seguir trabajando en un marco conceptual para la gamificación (ludificación) basado en elementos de motivación, resultados psicológicos, resultados conductuales y efectos en y desde el neuroaprendizaje para ser aplicados en la teoría del aprendizaje en primera infancia. Es necesario plantear estrategias metodológicas para la incorporación de la gamificación o ludificación en contextos de infancias con el fin de ser evaluados y validados por la comunidad disciplinar, ya que se evidencian numerables aplicaciones, pero ninguna de ellas ofrece una secuencia de aprendizaje replicable en diversidad de contextos.

Además, es importante definir el papel del contexto en un espacio a implementar la gamificación o ludificación. Se debe continuar con la revisión de la literatura a través de una metodología rigurosa que permita explorar otras conceptualizaciones y/o aproximaciones epistemológicas que, desde los campos de la infancia, la cibercultura y la transmedia se realicen para el objeto de estudio. Se deben superar limitaciones metodológicas en la formulación de investigaciones y/o estudios en gamificación (ludificación), tales como:

1. los tamaños de las muestras son pequeños en la mayoría de estudios

2. Utilización de mediciones psicométricas para componentes de motivación y conducta.

3. Se debe contar con grupos de control que validen las implementaciones en grupos focales. 
4. Establecer relaciones entre procesos cognitivos, emociones, reto y logro

5. Presentar plazos de investigación más amplios que permitan realizar correlaciones de resultados.

En cuanto a las limitaciones de la presente revisión de la literatura, se encuentra que es un tema de estudio joven y pocos son los documentos que en idioma español se encuentran en las bases de datos, marcos teóricos o comunicaciones a eventos científicos. Estas situaciones limitaron la investigación desde lo conceptual y teórico; por tal razón, es importante este hallazgo en cuanto se crea la necesidad latente de investigar, sistematizar y conceptualizar procesos de gamificación o ludificación en distintos contextos.

Finalmente, los artículos revisados coinciden en el potencial de la gamificaciónludificación en procesos de conducta y aprendizaje; se hace referencia directa de este proceso en la totalidad de ellos, en los cuales se presentan las posibilidades de mejora en el aprendizaje de estudiantes a través de la gamificación-ludificación de actividades escolares y/o el uso de estrategias de videojuegos o TIC. Asimismo, no se evidencio ningún artículo que hable de las oportunidades que puede tener el desarrollo de aprendizajes y/o procesos cognitivos en la primera infancia desde la ludificación y la gamificación.

Por tal razón, el acercamiento al estudio de las perspectivas teóricas evidenciadas en los artículos revisados, permitió identificar que la integración de la gamificaciónludificación en los procesos de aprendizaje en la primera infancia está conformada por una serie de variables, entre las que se encuentran las áreas disciplinares, corrientes del pensamiento, teorías educativas, dinámicas de gamificación y experiencias investigativas que permitan entender y comprender como se fundamenta una investigación en el tema de la gamificación y la primera infancia.

Con el análisis de documentos y revisión de fuentes bibliográficas, se puede concluir que son suficientes las experiencias que se encuentran sobre ludificación y primera infancia que están sustentadas en proceso de investigación rigurosos y con un mínimo de tiempo experimental. Sin embargo, esta existencia exponencial de artículos, en los últimos cuatro años, sobre investigaciones en ludificación y primera infancia permite concluir que no se realicen ejercicios de este tipo en las aulas y centros educativos para la primera infancia, sino que conduce la mirada a la conclusión realizada por Lee 
(2011), donde habla que la ludificación aplicada a la educación se maneja en una escala propia de aplicación, en primer lugar se encuentran profesores que a título propio aplican innovaciones en el aula, tales como sustituir notas tradicionales por puntos o insignias; organizar ejercicios extracurriculares a través de tableras de insignias, generación de actividades retadoras y complementarias que generan motivaciones extrínsecas e intrínsecas sobre algún tema específico del currículo, o con el uso e integración, de las TIC, la informática y los medios de comunicación en la coyuntura y desarrollo de la clase.

Por otro lado, se encuentran instituciones que asumen características, componentes y estrategias de juego para el diseño de sus planes de estudio; sin embargo, en ambos casos no se es consciente por los autores la integración de la ludificación al proceso, además de no estar documentado el ejercicio y no estar sustentado en un proceso de investigación. Esta situación ayuda a comprender mejor la gamificación, comprendiendo que ludificación en el aprendizaje es mas allá de llevar TIC y/o usar un juego / videojuego y nos sitúa en comprender la integración de la ludificación en entornos de aprendizaje para la primera infancia debe saber integrar tres conceptos esenciales, tal y como lo reconoce Hunicke (2013), las mecánicas, las dinámicas y las estéticas del juego.

De esta forma, se entienden las mecánicas de juego en el aprendizaje de la primera infancia como los componentes más importantes de un juego, estos determinan las lógicas del juego, los propósitos y las ideas de aprendizaje que propone el juego, además de clarificar las funcionalidades ejecutivas y el desarrollo cognitivo, social y emocional que fomentara el juego. Las dinámicas de juego en la primera infancia se encargan de definir el comportamiento de las distintas mecánicas de juego, en cuanto la interacción, el trabajo cooperativo, pero también, la motricidad, las funcionalidades visual y auditiva, los circuitos neuromotores, la emoción, la emotividad, el reto y el logro, la frustración, la dificultad; por último, las estéticas de juego en la primera infancia son aquellas en las que se definen las respuestas emocionales y cognitivas que se espera despertar en el niño y la niña (Pineda y Orozco, 2018, p. 160).

Lo anterior, nos permite concluir que existe una latente relación entre ludificación y primera infancia y, que esta relación está sustentada en las variables de motivación, conducta, aprendizaje y juego. Asimismo, dicha relación propende la mejora de procesos cognitivos y conductuales de base neuropsicología y de funciones ejecutivas de niños y 
niñas. Por tanto, se cree que esta el camino listo para iniciar la etapa de intervención del proyecto, el cual está enfocado en la construcción y diseño de un modelo de aprendizaje ludificado para la primera infancia.

\section{Referencias bibliográficas}

Acuña, L.F. (2008). El estado del arte del concepto y abordaje pedagógico de las dificultades de aprendizaje en la Ciudad de Bogotá en los últimos siete años. Bogotá, Colombia: IDEP.

Blanco, Y., Zea, L.A., Espitia, G., Acuña, L.A., (2013). Estudio Valoración Abordaje de procesos de desarrollo, aprendizaje y sus dificultades aplicando un instrumento de valoración de niños y niñas para ciclo inicial en tres instituciones de la Ciudad de Bogotá en Innovar en la Escuela. Bogotá, Colombia: IDEP.

República de Colombia. (2006). Código de la Infancia y la Adolescencia

Ermi, L., Mäyrä, F. (2005). Player-Centred Game Design: Experiences in Using Scenario Study to Inform Mobile Game Design. Game Studies, 5, no. 1.

Hamari, J., Koivisto, J., \& Pakkanen, T. (2014). Do Persuasive Technologies Persuade? A Review of Empirical Studies. In: Spagnol

Hunicke R. (2013). MDA: A Formal Approach to Game Design and Game Research. Dominguez,

Webster, J. Watson, R.T. (2002). Analyzing the past to prepare for the future: writing a literature review, MIS Quarterly, 26(2), xiii-xxiii.

Johnson, S. (2012). Cultura basura, cerebros privilegiados. Barcelona, España: Roca Editorial.

Juliao, C. (2002). La praxeología: una teoría de la práctica. Bogotá, Colombia: UNIMINUTO.

Juliao, C. (2011). El enfoque praxeológico. Bogotá, Colombia: Corporación Universitaria Minuto de Dios. UNIMINUTO.

Kapp, K. (2012). The Gamification of Learning and Instruction: Game-Based Methods and Strategies for Training and Education. San Francisco: John Wiley \& Sons. 
Lee, J.; Ceyhan, P.; Jordan-Cooley, W. y Sung, W. (2013) GREENIFY: A Real-World Action Game for Climate Change Education. Simulation \& Gaming, Paper. Disponible en: http://tcgameslab.org/wp-content/uploads/2013/02/Lee-et-al.Greenify-Simulationand-Gaming-2013.pdf

Lee, J. y Hammer, J. (2011). Gamification in education: What, how, why bother? Academic Exchange Quarterly, volumen 15, 1-5.

Orozco, P.; Pineda, E. (2017). Investigación educativa desde la perspectiva de la pedagogía praxeológica. Villavicencio: Corporación Universitaria Minuto de Dios UNIMINUTO.

Pelegrina del Río, M.; Tejeiro Salguero, R. (2008). La psicología de los videojuegos: un modelo de investigación. Málaga, España: Aljibe.

Pineda, E; Velásquez, O. (2015). La Sistematización de experiencias desde el enfoque praxeológico. Villavicencio: Corporación Universitaria Minuto de Dios UNIMINUTO.

Pineda, E; Orozco, P. (2018 a). Estado del arte y abordaje del concepto de ludificación en el aprendizaje en primera infancia. Infancias Imágenes, 17(2), 147-162. https://doi.org/10.14483/16579089.12471

Pineda, E.; Orozco, P. (2018b). Lineamientos generales para la reorganización de la educación desde las pedagogías críticas. Villavicencio: Ediciones USTA.

Pineda, E.; Orozco, P. (2018c). Herramientas digitales para la investigación educativa. Villavicencio: Ediciones USTA.

Pineda, E.; Orozco, P. (2017). Neuropsicología y aprendizaje infantil ludificado. Una alternativa pedagógica. Madrid: Editorial Académica Española.

Pineda, E. Cabrera, J. Cuervo, N.R (2014). Linea base de oferta y agentes educativos en primera infancia en los 120 municipios no certificados del departamento de Boyacá. Tunja, Colombia: Ascender.

Shotton, M. (1992) Computer adiction?: A study of computer dependency. Londres, Inglaterra: Taylor and Francis.

Tejeiro, R.; Pelegrina del Río, M. y Vallecillo, M. (2009). Efectos psicosociales de los videojuegos. Comunicación, 1, 235 -250. 
UNICEF (1999). Declaración Universal de los Derechos del Niño. Ginebra, Suiza: UNICEF.

Vassileva, J. (2012). Motivating Participation in Social Computing Applications: A User Modeling Perspective. User Modeling and User-Adapted Interaction, 22, 177201.

Zichermann, G. y Cunningham, C. (2011). Gamification by Design: Implementing Game Mechanics in Web and Mobile Apps. Cambridge, MA: O'Reilly Media. 